Sādhanā Vol. 39, Part 1, February 2014, pp. 189-206. (C) Indian Academy of Sciences

\title{
A new method for solving single and multi-objective fuzzy minimum cost flow problems with different membership functions
}

\author{
AMIT KUMAR $^{1}$ and MANJOT KAUR ${ }^{2, *}$ \\ ${ }^{1}$ School of Mathematics and Computer Applications, Thapar University, Patiala, \\ 147004 , India \\ ${ }^{2}$ Department of Mathematics, Dr BR Ambedkar National Institute of Techonology, \\ Jalandhar 144011, India \\ e-mail: amit_rs_iitr@yahoo.com; manjot.thaparian@gmail.com
}

MS received 15 October 2012; revised 29 August 2013; accepted 29 August 2013

\begin{abstract}
Several authors have proposed different methods for solving fuzzy minimum cost flow (MCF) problems. In this paper, some single and multi-objective fuzzy MCF problems are chosen which cannot be solved by using any of the existing methods and a new method is proposed for solving such type of problems. The main advantage of the proposed method over existing methods is that the fuzzy MCF problems which can be solved by using the existing methods can also be solved by the proposed method. But, there exist several fuzzy MCF problems which can be solved only by using the proposed method i.e., it is not possible to solve these problems by using the existing methods. To illustrate the proposed method and also to show the advantages of the proposed method over existing methods some single and multiobjective fuzzy MCF problems which cannot be solved by using the existing methods are solved by using the proposed method and the obtained results are discussed.
\end{abstract}

Keywords. Single and multi-objective fuzzy MCF problems; fuzzy linear programming; $L-R$ fuzzy numbers; ranking function.

\section{Introduction}

MCF problem is a general form of the network flow problem whose aim is to find the least cost of the shipment of a commodity through a capacitated network in order to satisfy demands at certain nodes from available supplies at other nodes. Because it represents a general form of the network flow, the results from the study of the MCF problem can be applied to many other network problems such as transportation, maximum flow, assignment, shortest path, and transshipment problems. The MCF problem is also very practical, it has been used to solve several

*For correspondence 
real-world applicational problems such as multi-stage production inventory planning, mold allocation, nurse scheduling, project assignment, faculty course assignment, and automobile routing (Ahuja et al 1993).

The MCF can be formulated as a linear programming where the constraints have a special structure. In classical form, the MCF problem minimizes the cost of transporting some product that is available at some sources and required at some destinations. However, in most real world problems due to the complexity of the social and economic environment there may also be need to consider the explicit objective functions other than cost. These objectives are frequently in conflict, measured in different scales and difficult to combine in one overall utility function. Multi-objective MCF problems have been significantly studied in the literature (Hamacher et al 2007).

In actual practice, the costs and the capacities of the network are generally vague or uncertain. Fuzzy set theory (Zadeh 1965) appears to be ideally suited to solve such vague aspects. Shih and Lee (Shih \& Lee 1999) proposed a fuzzy version of MCF problems by using multi-level linear programming problem.

Liu \& Kao (2004) solved MCF problems with fuzzy costs using Yager's ranking index which maintains the network structure of the problem which permits to use network simplex method. Ghatee \& Hashemi (2008) presented some different cases of fuzzy MCF problems utilizing a total order and nominal flows. Ghatee \& Hashemi (2007) investigated fully fuzzified MCF problems considering a large variety of ranking functions. Ghatee et al (2008) introduced the duality properties for fully fuzzified version of the MCF problem. Ghatee et al (2009) introduced a method for solving MCF problems with fuzzy costs. The application of fuzzy MCF problems in internet transmission (Liu \& Kao 2004) petroleum industry (Ghatee \& Hashemi 2007) and bus network planning (Ghatee \& Hashemi 2008) have been also presented in recent literature. Ghatee and Hashemi (Ghatee \& Hashemi 2009) studied uncertainty and impreciseness, multiple objectives and optimistic and pessimistic attitudes, dealing with a traditional MCF problem and its application in network design.

In this paper, some single and multi-objective fuzzy MCF problems are chosen which cannot be solved by using any of the existing methods and a new method is proposed for solving such type of problems. The main advantage of the proposed method over existing methods is that the fuzzy MCF problems which can be solved by using the existing methods can also be solved by proposed method. But, there exist several fuzzy MCF problems which can be solved only by using the proposed method i.e., it is not possible to solve these problems by using the existing methods. To illustrate the proposed method and also to show the advantages of the proposed method over existing methods some single and multi-objective fuzzy MCF problems which cannot be solved by using the existing methods are solved by using the proposed method and the obtained results are discussed.

This paper is organized as follows: In section 2, some basic definitions and Yager's ranking index for the ranking of fuzzy numbers are presented. In section 3, linear programming formulation of multi-objective MCF problems in crisp and fuzzy environment is presented. In section 4, limitations of existing methods for solving fuzzy MCF problems are discussed. In section 5, fuzzy programming technique to solve multi-objective problems is presented. In section 6 , a new method is proposed to find the solution of single and multi-objective fuzzy MCF problems. In section 7, advantages of the proposed method over existing method are presented. Also, to illustrate the proposed method and to show the advantage of proposed method over existing methods (Shih \& Lee 1999; Liu \& Kao 2004; Ghatee et al 2009) some single and multi-objective fuzzy MCF problems which cannot be solved by using the existing methods are solved. The obtained results are discussed in section 8 . The conclusions are provided in section 9. 


\section{Preliminaries}

In this section some basic definitions and Yager's ranking index for the ranking of fuzzy numbers are presented.

\subsection{Basic definitions}

In this section, some basic definitions are presented (Dubois \& Prade 1980)

Definition 1 A fuzzy number $\tilde{A}=(m, n, \alpha, \beta)_{L R}$ is said to be a $L-R$ flat fuzzy number if

$$
\mu_{\tilde{A}}(x)= \begin{cases}L\left(\frac{m-x}{\alpha}\right), & \text { for } x \leq m, \alpha>0 \\ R\left(\frac{x-n}{\beta}\right), & \text { for } x \geq n, \beta>0 \\ 1, & \text { otherwise }\end{cases}
$$

If $m=n$ then $\tilde{A}=(m, n, \alpha, \beta)_{L R}$ will be converted into $\tilde{A}=(m, \alpha, \beta)_{L R}$ and is said to be an $L-R$ fuzzy number.

$L$ and $R$ are called reference functions, which are continuous, non-increasing functions that defining the left and right shapes of $\mu_{\tilde{A}}(x)$, respectively and $L(0)=R(0)=1$. Two special cases are triangular and trapezoidal fuzzy number, for which $L(x)=R(x)=\operatorname{maximum}\{0,1-|x|\}$, are linear functions. Three commonly used nonlinear reference functions with parameters $q$, denoted as $R F_{q}$, are summarized as follows:

Power: $R F_{q}(x)=$ maximum $\left\{0,1-|x|^{q}\right\}, q \geq 0$,

Exponential power: $R F_{q}(x)=e^{-|x|^{q}}, q \geq 0$,

Rational: $R F_{q}(x)=\frac{1}{\left(1+|x|^{q}\right)}, q \geq 0$.

Definition 2 Let $\tilde{A}=(m, n, \alpha, \beta)_{L R}$ be a $L-R$ flat fuzzy number and $\lambda$ be a real number in the interval $[0,1]$ then the crisp set $A_{\lambda}=\left\{x \in X: \mu_{\tilde{A}}(x) \geq \lambda\right\}=$ $\left[m-\alpha L^{-1}(\lambda), n+\beta R^{-1}(\lambda)\right]$ is said to be $\lambda-$ cut of $\tilde{A}$.

\subsection{Yager's ranking approach}

A number has been proposed for the ranking of fuzzy numbers. A relatively simple computational and easily understandable ranking approach proposed by Yager (1981) is considered for the ranking of fuzzy numbers in this paper. Yager (1981) proposed a procedure for ordering fuzzy sets in which a ranking index $I(\tilde{A})$ is calculated for the fuzzy number $\tilde{A}=$ $(m, n, \alpha, \beta)_{L R}$ from its $\lambda-$ cut $A_{\lambda}=\left\{x \in X: \mu_{\tilde{A}}(x) \geq \lambda\right\}=\left[m-\alpha L^{-1}(\lambda), n+\beta R^{-1}(\lambda)\right]$ according to the following formula:

$$
I(\tilde{A})=\frac{1}{2}\left(\int_{0}^{1}\left(m-\alpha L^{-1}(\lambda)\right) d \lambda+\int_{0}^{1}\left(n+\beta R^{-1}(\lambda)\right) d \lambda\right) .
$$

Since it is calculated for the convex fuzzy number $\tilde{A}$ from the extreme values of its $\lambda$ - cut, $m-\alpha L^{-1}(\lambda)$ and $n+\beta R^{-1}(\lambda)$, rather than its membership function, it is not required $\alpha$ to know the explicit form of the membership functions of the fuzzy numbers to be ranked. That is, unlike most of the ranking methods that requires the knowledge of the membership functions of 
all fuzzy numbers to be ranked, the Yager's ranking index is still applicable even if the explicit form of the membership function of the fuzzy number is unknown.

Let $\tilde{A}$ and $\tilde{B}$ be two fuzzy numbers then

(i) $\tilde{A}>_{I} \tilde{B}$ if $I(\tilde{A})>I(\tilde{B})$,

(ii) $\tilde{A}={ }_{I} \tilde{B}$ if $I(\tilde{A})=I(\tilde{B})$,

(iii) $\tilde{A} \geq_{I} \tilde{B}$ if $I(\tilde{A}) \geq I(\tilde{B})$.

2.2a Linearity property of Yager's ranking index: Let $\tilde{A}=\left(m_{1}, n_{1}, \alpha_{1}, \beta_{1}\right)_{L_{1}-R_{1}}$ and $\tilde{B}=$ $\left(m_{2}, n_{2}, \alpha_{2}, \beta_{2}\right)_{L_{2}-R_{2}}$ be two $L-R$ flat fuzzy numbers and $k_{1}, k_{2}$ be two non-negative real numbers.

Using Definition 2, the $\lambda-$ cut $A_{\lambda}$ and $B_{\lambda}$ corresponding to $\tilde{A}$ and $\tilde{B}$ are

$$
A_{\lambda}=\left[m_{1}-\alpha_{1} L_{1}^{-1}(\lambda), n_{1}+\beta_{1} R_{1}^{-1}(\lambda)\right] \text { and } B_{\lambda}=\left[m_{2}-\alpha_{2} L_{2}^{-1}(\lambda), n_{2}+\beta_{2} R_{2}^{-1}(\lambda)\right]
$$

Using the property, $\left(\delta_{1} A_{1}+\delta_{2} A_{2}\right)_{\lambda}=\delta_{1}\left(A_{1}\right)_{\lambda}+\delta_{2}\left(A_{2}\right)_{\lambda}, \forall \delta_{1}, \delta_{2} \in R$ ( $R$ is a set of real numbers), the $\lambda-\operatorname{cut}\left(k_{1} A+k_{2} B\right)_{\lambda}$ corresponding to $\left(k_{1} \tilde{A} \oplus k_{2} \tilde{B}\right)$ is

$\left(k_{1} A+k_{2} B\right)_{\lambda}$

$$
=\left[k_{1} m_{1}+k_{2} m_{2}-k_{1} \alpha_{1} L_{1}^{-1}(\lambda)-k_{2} \alpha_{2} L_{2}^{-1}(\lambda), k_{1} n_{1}+k_{2} n_{2}+k_{1} \beta_{1} R_{1}^{-1}(\lambda)+k_{2} \beta_{2} R_{2}^{-1}(\lambda)\right] .
$$

Using section 2.2, the Yager's ranking index $I\left(k_{1} \tilde{A} \oplus k_{2} \tilde{B}\right)$ corresponding to fuzzy number $\left(k_{1} \tilde{A}+k_{2} \tilde{B}\right)$ is

$$
\begin{aligned}
I\left(k_{1} \tilde{A} \oplus k_{2} \tilde{B}\right)= & \frac{1}{2} k_{1}\left[\int_{0}^{1}\left(m_{1}-\alpha_{1} L_{1}^{-1}(\lambda)\right) d \lambda+\int_{0}^{1}\left(n_{1}+\beta_{1} R_{1}^{-1}(\lambda)\right) d \lambda\right]+ \\
& \frac{1}{2} k_{2}\left[\int_{0}^{1}\left(m_{2}-\alpha_{2} L_{2}^{-1}(\lambda)\right) d \lambda+\int_{0}^{1}\left(n_{2}+\beta_{2} R_{2}^{-1}(\lambda)\right) d \lambda\right] \\
= & k_{1} I(\tilde{A})+k_{2} I(\tilde{B}) .
\end{aligned}
$$

Similarly, it can be proved that $I\left(k_{1} \tilde{A} \oplus k_{2} \tilde{B}\right)=k_{1} I(\tilde{A})+k_{2} I(\tilde{B}) \quad \forall k_{1}, k_{2} \in R$.

\section{Linear programming formulation of multi-objective MCF problems in crisp and fuzzy environment}

In this section, the linear programming formulations of multi-objective MCF problems in crisp and fuzzy environment are presented (Shih \& Lee 1999). 


\subsection{Linear programming formulation of multi-objective MCF problems in crisp environment}

If all the parameters of the multi-objective MCF problem are represented by real numbers then any multi-objective MCF problem may be formulated into the following crisp linear programming problem (Shih \& Lee 1999).

$$
\text { Minimize } \sum_{(i, j) \in A} c_{i j}^{\eta} x_{i j} \quad ; \eta=1,2, \ldots, P
$$

subject to

$$
\begin{aligned}
\sum_{j:(i, j) \in A} x_{i j}-\sum_{k:(k, i) \in A} x_{k i}=b(i) & ; \forall i \in V, \\
x_{i j} \in U_{i j} & ; \forall(i, j) \in A, \\
x_{i j} \geq 0 & ; \forall(i, j) \in A .
\end{aligned}
$$

where, $A$ : The set of $\operatorname{arcs}(i, j)$,

$V$ : Set of nodes,

$x_{i j}$ : The decision variable denoting the flow through arc $(i, j)$,

$U_{i j}=\left[l_{i j}, u_{i j}\right]$ : Capacity for $\operatorname{arc}(i, j)$,

$c_{i j}^{\eta}$ : The penalty per unit of flow through arc $(i, j)$ in the $\eta^{\text {th }}$ objective function $\eta=$ $1,2, \ldots, P$, where $P$ is total number of objective functions.

$b(i)$ : The net flow generated at node $i$, The value of $b(i)$ being positive, zero or negative classifies node $i$ as a supply node, transshipment node, or demand node, respectively.

\subsection{Linear programming formulation of multi-objective MCF problems in fuzzy environment}

If the parameters $c_{i j}^{\eta}$ and $U_{i j}$ are represented by fuzzy numbers $\tilde{c}_{i j}^{\eta}$ and $\tilde{U}_{i j}$ then any multiobjective MCF problem may be formulated into the following fuzzy linear programming the problem (Shih \& Lee 1999).

$$
\text { Minimize } \sum_{(i, j) \in A} \tilde{c}_{i j}^{\eta} x_{i j} \quad ; \eta=1,2, \ldots, P
$$

subject to

$$
\begin{aligned}
\sum_{j:(i, j) \in A} x_{i j}-\sum_{k:(k, i) \in A} x_{k i}=b(i) & ; \forall i \in V, \\
x_{i j} \in \tilde{U}_{i j} & ; \forall(i, j) \in A, \\
x_{i j} \geq 0 & ; \forall(i, j) \in A .
\end{aligned}
$$

where, $\tilde{c}_{i j}=\left(c_{i j}^{\prime}, c_{i j}^{\prime \prime}, \alpha_{i j}^{\prime}, \beta_{i j}^{\prime}\right)_{L R}$ and $\tilde{U}_{i j}=\left(u_{i j}^{\prime}, u_{i j}^{\prime \prime}, \alpha_{i j}^{\prime \prime}, \beta_{i j}^{\prime \prime}\right)_{L R}$

Remark 3.1. If $p=1$ then the problem $\left(P_{1}\right)$ will represent a single objective fuzzy MCF problem.

\section{Limitations of existing methods}

Shih \& Lee (1999) proposed a new method which can be used to solve single and multi-objective fuzzy MCF problems. Liu \& Kao (2004) and Ghatee et al (2009) pointed out the shortcomings of the existing method (Shih \& Lee 1999) and proposed a new method for solving the same type of single objective fuzzy MCF problems. 
In this section, the limitations of all the existing methods (Shih \& Lee 1999; Liu \& Kao 2004; Ghatee et al 2009) are pointed out.

The existing methods (Shih \& Lee 1999; Liu \& Kao 2004; Ghatee et al 2009) can be used only to find the solution of such single and multi-objective fuzzy MCF problems in which all the uncertain penalty parameters (i.e., cost, time, etc.) are represented by same type of $L-R$ fuzzy numbers i.e., it can be used only for solving the fuzzy MCF problems $\left(P_{1}\right)$.

Example 4.1. The fuzzy MCF problem (Ghatee et al 2009) may be formulated into the following fuzzy linear programming:

$$
\begin{aligned}
& \text { Minimize }\left(\begin{array}{l}
(40,35,50)_{L R} x_{13} \oplus(45,41,70)_{L R} x_{14} \oplus(57,52,59)_{L R} x_{15} \oplus \\
(40,30,45)_{L R} x_{23} \oplus(50,40,70)_{L R} x_{24} \oplus(60,47,104)_{L R} x_{25} \oplus \\
(10,8,15)_{L R} x_{34} \oplus(50,42,95)_{L R} x_{36} \oplus(35,33,37)_{L R} x_{37} \oplus \\
(10,7,30)_{L R} x_{45} \oplus(40,32,75)_{L R} x_{46} \oplus(25,20,31)_{L R} x_{47} \oplus \\
(30,25,45)_{L R} x_{56} \oplus(10,8,30)_{L R} x_{57}
\end{array}\right) \\
& \text { subject to } \\
& x_{13}+x_{14}+x_{15}=30 \\
& x_{23}+x_{24}+x_{25}=60 \\
& x_{34}+x_{36}+x_{37}-x_{13}-x_{23}=0 \\
& x_{45}+x_{46}+x_{46}-x_{14}-x_{24}-x_{34}=0 \\
& x_{56}+x_{57}-x_{15}-x_{25}-x_{45}=0 \\
& -x_{36}-x_{46}-x_{56}=-20 \\
& -x_{37}-x_{47}-x_{57}=-70 \\
& x_{13} \in[0,40], x_{14} \in[0,10], x_{15} \in[0,15], x_{23} \in[0,50], x_{24} \in[0,80], x_{25} \in[0,40], \\
& x_{34} \in[0,20], x_{36} \in[0,100], x_{37} \in[0,90], x_{45} \in[0,15],, x_{46} \in[0,30], x_{47} \in[0,20], \\
& x_{56} \in[0,15], x_{57} \in[0,100] .
\end{aligned}
$$

where, $L(x)=R(x)=\operatorname{maximum}\{0,1-|x|\}$

Several authors (Mon \& Cheng 1994; Chanas \& Kuchta 1998; Chen 2007; Chen \& Hsueh 2008; Kumar et al 2008; Ching \& Cheng 2009; Zareei et al 2011) have pointed out that there may exist several real life problems in which it is not possible to represent the different parameters by same type of $L-R$ fuzzy numbers and to overcome this short coming they have represented the different parameters by different type of $L-R$ fuzzy numbers. There may also exist several single and multi-objective MCF problems in which there is need to represent different uncertain penalty parameters by different type of $L-R$ fuzzy numbers. The existing methods (Shih \& Lee 1999; Liu \& Kao 2004; Ghatee et al 2009) cannot be used for solving the following such type of single and multi-objective fuzzy MCF problems e.g., the existing methods (Shih \& Lee 1999; Liu \& Kao 2004; Ghatee et al 2009) cannot be used to solve the following type of single and multi-objective fuzzy MCF problems:

$$
\begin{array}{cl}
\text { Minimize } \sum_{(i, j) \in A} \tilde{c}_{i j}^{\eta} x_{i j} & ; \eta=1,2, \ldots, P \\
\text { subject to } & ; \forall i \in V, \\
\sum_{j:(i, j) \in A} x_{i j}-\sum_{\substack{k:(k, i) \in A \\
x_{i j} \in \tilde{U}_{i j}}} x_{k i}=b(i) & ; \forall(i, j) \in A \\
x_{i j} \geq 0 & ; \forall(i, j) \in A .
\end{array}
$$


where,

$$
\tilde{c}_{i j}=\left(c_{i j}^{\prime}, c_{i j}^{\prime \prime}, \alpha_{i j}^{\prime}, \beta_{i j}^{\prime}\right)_{L_{i j}-R_{i j}} \text { and } \tilde{U}_{i j}=\left(u_{i j}^{\prime}, u_{i j}^{\prime \prime}, \alpha_{i j}^{\prime \prime}, \beta_{i j}^{\prime \prime}\right)_{L_{i j}-R_{i j}}
$$

Example 4.2. Let us consider a fuzzy MCF problem with 5 nodes and 7 arcs as shown in figure 1. The fuzzy data for the chosen fuzzy MCF problem is summarized in tables 1-4.

The chosen fuzzy MCF problem may be formulated into the following single and multiobjective fuzzy linear programming problems:

Case (i): Considering only single objective i.e., to minimize the cost only, the chosen fuzzy MCF problem may be formulated into the following single objective fuzzy linear programming problem:

$\operatorname{Minimize}\left(\begin{array}{l}(2,3,1,2)_{L_{12}-R_{12}} x_{12} \oplus(5,5,1,1)_{L_{13}-R_{13}} x_{13} \oplus(7,8,2,5)_{L_{24}-R_{24}} x_{24} \\ \oplus(10,15,1,1)_{L_{25}-R_{25}} x_{25} \oplus(8,10,1,1)_{L_{34}-R_{34}} x_{34} \oplus(10,12,1,2)_{L_{35}-R_{35}} x_{35} \\ \oplus(4,6,2,2)_{L_{45}-R_{45}} x_{45}\end{array}\right)$

subject to

$$
\begin{aligned}
& x_{12}+x_{13}=10 \\
& x_{24}+x_{25}-x_{12}=0 \\
& x_{34}+x_{35}-x_{13}=20 \\
& x_{45}-x_{24}-x_{34}=-15 \\
& -x_{25}-x_{35}-x_{45}=-15 \\
x_{12} \in & (20,30,20,20)_{L_{12}-R_{12}}, x_{13} \in(10,30,10,30)_{L_{13}-R_{13}}, x_{24} \in(20,60,20,20)_{L_{24}-R_{24}} \\
x_{25} \in & (10,40,10,10)_{L_{25}-R_{25}}, x_{34} \in(30,60,30,30)_{L_{34}-R_{34}}, x_{35} \in(25,50,25,25)_{L_{35}-R_{35}} \\
x_{45} \in & (50,60,50,35)_{L_{45}-R_{45}} .
\end{aligned}
$$

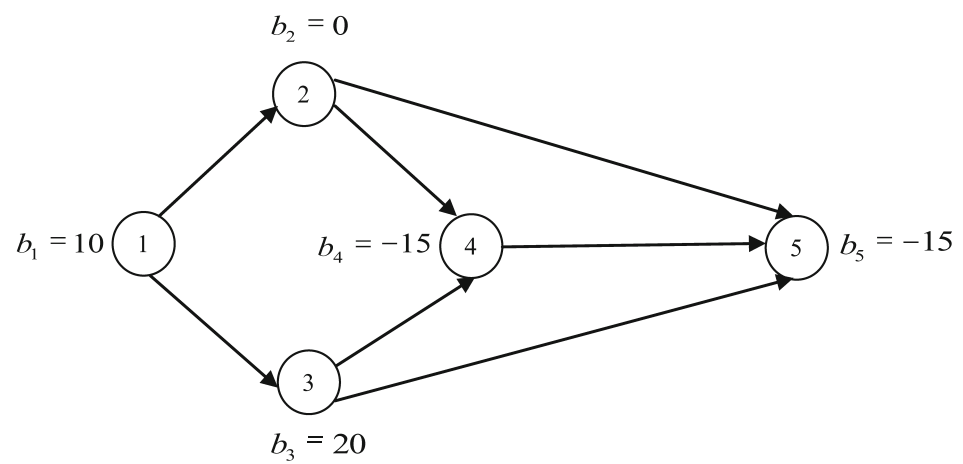

Figure 1. A network with 5 nodes and 7 arcs. 
Table 1. Parameters for a fuzzy MCF problem (with 5 nodes and 7 arcs).

\begin{tabular}{lcccc}
\hline $\begin{array}{l}\text { Node } \\
\text { no. }\end{array}$ & $\begin{array}{c}\text { Supply/ } \\
\text { demand }\end{array}$ & $\begin{array}{c}\text { Fuzzy cost } \\
\left(\tilde{c}_{i j}\right)\end{array}$ & $\begin{array}{c}\text { Fuzzy time } \\
\left(\tilde{t}_{i j}\right)\end{array}$ & $\begin{array}{c}\text { Fuzzy capacity } \\
\left(\tilde{U}_{i j}\right)\end{array}$ \\
\hline 1 & 10 & $\tilde{c}_{12}=(2,3,1,2)_{L_{12}-R_{12}}$ & $\tilde{t}_{12}=(9,9,1,1)_{L_{12}-R_{12}}$ & $(20,30,20,20)_{L_{12}-R_{12}}$ \\
2 & 0 & $\tilde{c}_{13}=(5,5,1,1)_{L_{13}-R_{13}}$ & $\tilde{t}_{13}=(3,4,1,2)_{L_{13}-R_{13}}$ & $(10,30,10,30)_{L_{13}-R_{13}}$ \\
3 & 20 & $\tilde{c}_{24}=(7,8,2,5)_{L_{24}-R_{24}}$ & $\tilde{t}_{24}=(3,3,1,1)_{L_{24}-R_{24}}$ & $(20,60,20,20)_{L_{24}-R_{24}}$ \\
4 & -15 & $\tilde{c}_{25}=(10,15,1,1)_{L_{25}-R_{25}}$ & $\tilde{t}_{25}=(2,3,1,2)_{L_{25}-R_{25}}$ & $(10,40,10,10)_{L_{25}-R_{25}}$ \\
5 & -15 & $\tilde{c}_{34}=(8,10,1,1)_{L_{34}-R_{34}}$ & $\tilde{t}_{34}=(7,8,1,3)_{L_{34}-R_{34}}$ & $(30,60,30,30)_{L_{34}-R_{34}}$ \\
& & $\tilde{c}_{35}=(10,12,1,2)_{L_{36}-R_{36}}$ & $\tilde{t}_{35}=(6,9,2,3)_{L_{35}-R_{35}}$ & $(25,50,25,25)_{L_{35}-R_{35}}$ \\
& & $\tilde{c}_{45}=(4,6,2,2)_{L_{45}-R_{45}}$ & $\tilde{t}_{45}=(4,6,1,1)_{L_{45}-R_{45}}$ & $(50,60,50,35)_{L_{45}-R_{45}}$ \\
\hline
\end{tabular}

Case (ii): Considering only single objective i.e., to minimize the time only, the chosen fuzzy MCF problem may be formulated into the following single objective fuzzy linear programming problem:

$\operatorname{Minimize}\left(\begin{array}{l}(9,9,1,1)_{L_{12}-R_{12}} x_{12} \oplus(3,4,1,2)_{L_{13}-R_{13}} x_{13} \oplus(3,3,1,1)_{L_{24}-R_{24}} x_{24} \\ \oplus(2,3,1,2)_{L_{25}-R_{25}} x_{25} \oplus(7,8,1,3)_{L_{34}-R_{34}} x_{34} \oplus(6,9,2,3)_{L_{35}-R_{35}} x_{35} \\ \oplus(4,6,1,1)_{L_{45}-R_{45}} x_{45}\end{array}\right)$

subject to

$$
\begin{aligned}
& x_{12}+x_{13}=10 \\
& x_{24}+x_{25}-x_{12}=0 \\
& x_{34}+x_{35}-x_{13}=20 \\
& x_{45}-x_{24}-x_{34}=-15 \\
& -x_{25}-x_{35}-x_{45}=-15 \\
x_{12} \in & (20,30,20,20)_{L_{12}-R_{12}}, x_{13} \in(10,30,10,30)_{L_{13}-R_{13}}, x_{24} \in(20,60,20,20)_{L_{24}-R_{24}} \\
x_{25} \in & (10,40,10,10)_{L_{25}-R_{25}}, x_{34} \in(30,60,30,30)_{L_{34}-R_{34}}, x_{35} \in(25,50,25,25)_{L_{35}-R_{35}} \\
x_{45} \in & (50,60,50,35)_{L_{45}-R_{45}} .
\end{aligned}
$$

Table 2. Fuzzy cost $\left(\tilde{c}_{i j}\right)$, left shape function $L_{i j}(x)$ and right shape function $R_{i j}(x)$ for each arc.

\begin{tabular}{lcc}
\hline Fuzzy cost $\left(\tilde{c}_{i j}\right)$ & Left shape function $\left(L_{i j}(x)\right)$ & Right shape function $\left(R_{i j}(x)\right)$ \\
\hline$\tilde{c}_{12}=(2,3,1,2)_{L_{12}-R_{12}}$ & $L_{12}(x)=\operatorname{maximum}\left\{0,1-x^{4}\right\}$ & $R_{12}=e^{-|x|}$ \\
$\tilde{c}_{13}=(5,5,1,1)_{L_{13}-R_{13}}$ & $L_{13}(x)=\operatorname{maximum}\{0,1-|x|\}$ & $R_{13}=\operatorname{maximum}\left\{0,1-x^{4}\right\}$ \\
$\tilde{c}_{24}=(7,8,2,5)_{L_{24}-R_{24}}$ & $L_{24}=\operatorname{maximum}\left\{0,1-x^{2}\right\}$ & $R_{24}=\operatorname{maximum}\left\{0,1-x^{2}\right\}$ \\
$\tilde{c}_{25}=(10,15,1,1)_{L_{25}-R_{25}}$ & $L_{25}=e^{-|x|}$ & $R_{25}(x)=e^{-|x|}$ \\
$\tilde{c}_{34}=(8,10,1,1)_{L_{34}-R_{34}}$ & $L_{34}(x)=\operatorname{maximum}\{0,1-|x|\}$ & $R_{34}(x)=\operatorname{maximum}\{0,1-|x|\}$ \\
$\tilde{c}_{35}=(10,12,1,2)_{L_{36}-R_{36}}$ & $L_{35}=e^{-|x|}$ & $R_{35}(x)=\operatorname{maximum}\left\{0,1-x^{4}\right\}$ \\
$\tilde{c}_{45}=(4,6,2,2)_{L_{45}-R_{45}}$ & $L_{45}=e^{-|x|}$ & $R_{45}=e^{-x^{2}}$ \\
\hline
\end{tabular}


Table 3. Fuzzy time $\left(\tilde{t}_{i j}\right)$, left shape function $L_{i j}(x)$ and right shape function $R_{i j}(x)$ for each arc.

\begin{tabular}{lcc}
\hline Fuzzy time $\left(\tilde{t}_{i j}\right)$ & Left shape function $\left(L_{i j}(x)\right)$ & Right shape function $\left(R_{i j}(x)\right)$ \\
\hline$\tilde{t}_{12}=(9,9,1,1)_{L_{12}-R_{12}}$ & $L_{12}(x)=\operatorname{maximum}\left\{0,1-x^{4}\right\}$ & $R_{12}=e^{-|x|}$ \\
$\tilde{t}_{13}=(3,4,1,2)_{L_{13}-R_{13}}$ & $L_{13}(x)=\operatorname{maximum}\{0,1-|x|\}$ & $R_{13}=\operatorname{maximum}\left\{0,1-x^{4}\right\}$ \\
$\tilde{t}_{24}=(3,3,1,1)_{L_{24}-R_{24}}$ & $L_{24}(x)=\operatorname{maximum}\{0,1-|x|\}$ & $R_{24}(x)=\operatorname{maximum}\{0,1-|x|\}$ \\
$\tilde{t}_{25}=(2,3,1,2)_{L_{25}-R_{25}}$ & $L_{25}=e^{-|x|}$ & $R_{25}(x)=\operatorname{maximum}\{0,1-|x|\}$ \\
$\tilde{t}_{34}=(7,8,1,3)_{L_{34}-R_{34}}$ & $L_{34}(x)=\operatorname{maximum}\left\{0,1-x^{4}\right\}$ & $R_{34}=\operatorname{maximum}\left\{0,1-x^{2}\right\}$ \\
$\tilde{t}_{35}=(6,9,2,3)_{L_{35}-R_{35}}$ & $L_{35}=\operatorname{maximum}\left\{0,1-x^{2}\right\}$ & $R_{35}=e^{-x^{2}}$ \\
$\tilde{t}_{45}=(4,6,1,1)_{L_{45}-R_{45}}$ & $L_{45}(x)=\operatorname{maximum}\left\{0,1-x^{4}\right\}$ & $R_{45}(x)=\operatorname{maximum}\{0,1-|x|\}$ \\
\hline
\end{tabular}

Case (iii): Considering both the objectives simultaneously i.e., to minimize the cost and time both, the chosen fuzzy MCF problem may be formulated into the following multi-objective fuzzy linear programming problem:

$\operatorname{Minimize}\left(\begin{array}{l}(2,3,1,2)_{L_{12}-R_{12}} x_{12} \oplus(5,5,1,1)_{L_{13}-R_{13}} x_{13} \oplus(7,8,2,5)_{L_{24}-R_{24}} x_{24} \\ \oplus(10,15,1,1)_{L_{25}-R_{25}} x_{25} \oplus(8,10,1,1)_{L_{34}-R_{34}} x_{34} \oplus(10,12,1,2)_{L_{35}-R_{35}} x_{35} \\ \oplus(4,6,2,2)_{L_{45}-R_{45}} x_{45}\end{array}\right)$

$\operatorname{Minimize}\left(\begin{array}{l}(9,9,1,1)_{L_{12}-R_{12}} x_{12} \oplus(3,4,1,2)_{L_{13}-R_{13}} x_{13} \oplus(3,3,1,1)_{L_{24}-R_{24}} x_{24} \\ \bigoplus(2,3,1,2)_{L_{25}-R_{25}} x_{25} \oplus(7,8,1,3)_{L_{34}-R_{34}} x_{34} \oplus(6,9,2,3)_{L_{35}-R_{35}} x_{35} \\ \oplus(4,6,1,1)_{L_{45}-R_{45}} x_{45}\end{array}\right)$

subject to

$$
\begin{aligned}
& x_{12}+x_{13}=10 \\
& x_{24}+x_{25}-x_{12}=0 \\
& x_{34}+x_{35}-x_{13}=20 \\
& x_{45}-x_{24}-x_{34}=-15 \\
& -x_{25}-x_{35}-x_{45}=-15 \\
& x_{12} \in(20,30,20,20)_{L_{12}-R_{12}}, x_{13} \in(10,30,10,30)_{L_{13}-R_{13}} \\
& x_{24} \in(20,60,20,20)_{L_{24}-R_{24}}, x_{25} \in(10,40,10,10)_{L_{25}-R_{25}}, \\
& x_{34} \in(30,60,30,30)_{L_{34}-R_{34}}, x_{35} \in(25,50,25,25)_{L_{35}-R_{35}} \\
& x_{45} \in(50,60,50,35)_{L_{45}-R_{45}} .
\end{aligned}
$$

Table 4. Fuzzy capacity, left shape function $L_{i j}(x)$ and right shape function $R_{i j}(x)$ for each arc.

\begin{tabular}{lcc}
\hline Fuzzy capacity $\left(\tilde{U}_{i j}\right)$ & Left shape function $\left(L_{i j}(x)\right)$ & Right shape function $\left(R_{i j}(x)\right)$ \\
\hline$(20,30,20,20)_{L_{12}-R_{12}}$ & $L_{12}(x)=\operatorname{maximum}\left\{0,1-x^{4}\right\}$ & $R_{12}=\operatorname{maximum}\left\{0,1-x^{4}\right\}$ \\
$(10,30,10,30)_{L_{13}}-R_{13}$ & $L_{13}(x)=\operatorname{maximum}\{0,1-|x|\}$ & $R_{13}=\operatorname{maximum}\left\{0,1-x^{4}\right\}$ \\
$(20,60,20,20)_{L_{24}-R_{24}}$ & $L_{24}(x)=\operatorname{maximum}\{0,1-|x|\}$ & $R_{24}(x)=\operatorname{maximum}\{0,1-|x|\}$ \\
$(10,40,10,10)_{L_{25}-R_{25}}$ & $L_{25}=\operatorname{maximum}\left\{0,1-x^{2}\right\}$ & $R_{25}(x)=\operatorname{maximum}\{0,1-|x|\}$ \\
$(30,60,30,30)_{L_{34}}-R_{34}$ & $L_{34}(x)=\operatorname{maximum}\left\{0,1-x^{4}\right\}$ & $R_{34}=\operatorname{maximum}\left\{0,1-x^{2}\right\}$ \\
$(25,50,25,25)_{L_{35}-R_{35}}$ & $L_{35}=\operatorname{maximum}\left\{0,1-x^{2}\right\}$ & $R_{35}=\operatorname{maximum}\left\{0,1-x^{4}\right\}$ \\
$(50,60,50,35)_{L_{45}}-R_{45}$ & $L_{45}(x)=\operatorname{maximum}\left\{0,1-x^{4}\right\}$ & $R_{45}(x)=\operatorname{maximum}\{0,1-|x|\}$ \\
\hline
\end{tabular}


Since in all the fuzzy linear programming problems, obtained by considering the different cases of Example 4.2, the different fuzzy cost and different fuzzy time are represented by different types of $L-R$ fuzzy numbers so it is not possible to solve all the fuzzy linear programming problems i.e., the fuzzy MCF problems, chosen in Case (i), Case (ii) and Case (iii) of Example 4.2, by using the existing methods (Shih \& Lee 1999; Liu \& Kao 2004; Ghatee et al 2009).

\section{Fuzzy programming technique to solve multi-objective linear programming problems}

In this section, the fuzzy programming technique (Verma et al 1997) which is used in this paper for solving multi-objective problems is presented. The optimal compromise solution of the multiobjective problems may be obtained by using the following steps.

Step 1: Formulate the given multi-objective problem into the following multi-objective linear programming problem:

$$
\begin{aligned}
& \text { Minimize } \sum C^{\eta} X ; \eta=1,2, \ldots, P \\
& \text { subject to } A X \geq \text { or }=\text { or } \leq b \\
& X \geq 0 .
\end{aligned}
$$

Step 2: Solve the multi-objective problem, obtained in Step 1, as a single-objective problem $P$ times, by taking one of the objectives at a time, to find $P$ optimal solutions $X^{1}, X^{2}, \ldots, X^{P}$.

Step 3: Find the value of each objective function corresponding to the each optimal solution obtained in Step 2. Let the value of $i^{t h}$ objective function $Z_{i}$ corresponding to $j^{t h}$ optimal solution $X^{j}$ is denoted by $Z_{i}\left(X^{j}\right)$.

Step 4: Find $U_{i}=\operatorname{maximum}_{1 \leq j \leq P}\left\{Z_{i}\left(X^{j}\right)\right\} \quad \forall i=1,2, \ldots, P$

$$
L_{i}=\operatorname{minimum}_{1 \leq j \leq P}\left\{Z_{i}\left(X^{j}\right)\right\} \quad \forall i=1,2, \ldots, P .
$$

Step 5: Define the linear membership function $\mu^{i}\left(Z_{i}\right) \forall i=1,2, \ldots, P$

$$
\text { where, } \mu^{i}\left(Z_{i}\right)=\left\{\begin{array}{ll}
1, & \text { if } Z_{i} \leq L_{i} \\
1-\frac{Z_{i}-L_{i}}{U_{i}-L_{i}}, & \text { if } L_{i}<Z_{i}<U_{i} \\
0, & \text { if } Z_{i} \geq U_{i}
\end{array} .\right.
$$

Step 6: Using the linear membership functions, obtained in Step 5, convert the multi-objective linear programming problem, obtained in Step 2, into the following single objective crisp linear programming problem:

Maximize $\lambda^{\prime}$

subject to

$$
\begin{aligned}
& Z_{i}+\lambda^{\prime}\left(U_{i}-L_{i}\right) \leq U_{i}, i=1,2, \ldots, P, \\
& A X \geq \text { or }=\text { or } \leq b, \\
& X \geq 0 .
\end{aligned}
$$


Step 7: Solve the crisp linear programming problem, obtained in Step 6, to find the optimal compromise solution of the chosen multi-objective linear programming problem.

Step 8: Put the value of $X$, obtained from Step 7, in each objective function to find the optimal value of each objective function.

\section{Proposed method}

To overcome all the limitations of the existing methods discussed in section 4 , a new method is proposed for solving single and multi-objective fuzzy MCF problems in this section.

The solution of single and multi-objective fuzzy MCF problems may be obtained by using the following steps:

Step 1: Formulate the chosen single or multi-objective fuzzy MCF into the following fuzzy linear programming problem:

$$
\begin{aligned}
& \text { Minimize } \sum_{(i, j) \in A} \tilde{c}_{i j}^{\eta} x_{i j} \\
& \text { subject to } \\
& \sum_{j:(i, j) \in A} x_{i j}-\sum_{k:(k, i) \in A} x_{k i}=b(i) ; \forall i \in V, \\
& x_{i j} \in \tilde{U}_{i j} \quad ; \forall(i, j) \in A, \\
& x_{i j} \geq 0 ; \quad ; \forall(i, j) \in A \text {, }
\end{aligned}
$$

where,

$$
\tilde{c}_{i j}=\left(c_{i j}^{\prime}, c_{i j}^{\prime \prime}, \alpha_{i j}^{\prime \prime}, \beta_{i j}^{\prime}\right)_{L_{i j}-R_{i j}} \text { and } \tilde{U}_{i j}=\left(u_{i j}^{\prime}, u_{i j}^{\prime \prime}, \alpha_{i j}^{\prime \prime}, \beta_{i j}^{\prime \prime}\right)_{L_{i j}-R_{i j}}
$$

Step 2: Convert the fuzzy linear programming problem, obtained in Step 1, into the following crisp linear programming problem:

$$
\begin{gathered}
\text { Minimize } \sum_{(i, j) \in A} I\left(\tilde{c}_{i j}^{\eta}\right) x_{i j} \\
\text { subject to } \\
\qquad \begin{array}{c}
\sum_{j ;(i, j) \in A} x_{i j}-\sum_{k ;(k, i) \in A} x_{k i}=b(i) \quad ; \forall i \in V, \\
x_{i j} \in\left[m-\alpha L^{-1}(\lambda), n+\beta R^{-1}(\lambda)\right] \quad ; \forall(i, j) \in A, \\
x_{i j} \geq 0, \lambda \in[0,1] \quad ; \forall(i, j) \in A .
\end{array}
\end{gathered}
$$

Step 3: Solve the crisp linear programming problem, obtained in Step 2, to find the Yager's ranking index $\sum I\left(\tilde{c}_{i j}^{\eta}\right) x_{i j}$ corresponding to each fuzzy objective function by using any existing technique.

\section{Advantages of the proposed method over existing methods}

In this section, the advantages of the proposed method over existing methods (Shih \& Lee 1999; Liu \& Kao 2004; Ghatee et al 2009) are discussed. 
The main advantage of the proposed method over existing methods (Shih \& Lee 1999; Liu \& Kao 2004; Ghatee et al 2009) is that the existing methods (Shih \& Lee 1999; Liu \& Kao 2004; Ghatee et al 2009) can be used only to solve a particular type of single and multi-objective fuzzy MCF problems in which all the uncertain penalty parameters are represented by same type of $L-R$ fuzzy numbers i.e., the existing methods (Shih \& Lee 1999; Liu \& Kao 2004; Ghatee et al 2009) cannot be used to solve several single and multi-objective MCF problems in which the different uncertain penalty parameters are represented by different type of $L-R$ fuzzy numbers, while the proposed method can be used to solve both type of single and multi-objective fuzzy MCF problems i.e., single and multi-objective fuzzy MCF problems in which the different uncertain penalty parameters are either represented by the same type of $L-R$ fuzzy numbers or different type of $L-R$ fuzzy numbers.

\subsection{Solution of the chosen single and multi-objective fuzzy MCF problems}

To illustrate the proposed method and also to show the advantages of the proposed method over existing method, the single and multi-objective fuzzy MCF problems, chosen in section 4, which cannot be solved by the existing methods (Shih \& Lee 1999; Liu \& Kao 2004; Ghatee et al 2009) are solved.

7.1a Solution of the chosen single objective fuzzy MCF problem: The single objective fuzzy MCF problem, chosen in Case (i) of Example 4.2, may be solved by using the following steps of the proposed method:

Step 1: The fuzzy linear programming formulation of single-objective fuzzy MCF problem, chosen in Case (i) of Example 4.2 is

$\operatorname{Minimize}\left(\begin{array}{l}(2,3,1,2)_{L_{12}-R_{12}} x_{12} \oplus(5,5,1,1)_{L_{13}-R_{13}} x_{13} \oplus(7,8,2,5)_{L_{24}-R_{24}} x_{24} \\ \bigoplus(10,15,1,1)_{L_{25}-R_{25}} x_{25} \oplus(8,10,1,1)_{L_{34}-R_{34}} x_{34} \oplus(10,12,1,2)_{L_{35}-R_{35}} x_{35} \\ \oplus(4,6,2,2)_{L_{45}-R_{45}} x_{45}\end{array}\right)$ subject to

$$
\begin{aligned}
& x_{12}+x_{13}=10 \\
& x_{24}+x_{25}-x_{12}=0 \\
& x_{34}+x_{35}-x_{13}=20 \\
& x_{45}-x_{24}-x_{34}=-15 \\
& -x_{25}-x_{35}-x_{45}=-15 \\
& x_{12} \in(20,30,20,20)_{L_{12}-R_{12}}, x_{13} \in(10,30,10,30)_{L_{13}-R_{13}}, \\
& x_{24} \in(20,60,20,20)_{L_{24}-R_{24}} \\
& x_{25} \in(10,40,10,10)_{L_{25}-R_{25}}, x_{34} \in(30,60,30,30)_{L_{34}-R_{34}}, \\
& x_{35} \in(25,50,25,25)_{L_{35}-R_{35}} \\
& x_{45} \in(50,60,50,35)_{L_{45}-R_{45}} .
\end{aligned}
$$

Step 2: The single objective fuzzy linear programming problem, obtained in Step 1, may be converted into the following crisp linear programming problem:

$$
\operatorname{Minimize}\left(\begin{array}{l}
I\left((2,3,1,2)_{L_{12}-R_{12}}\right) x_{12}+I\left((5,5,1,1)_{L_{13}-R_{13}}\right) x_{13}+I\left((7,8,2,5)_{L_{24}-R_{24}}\right) x_{24} \\
+I\left((10,15,1,1)_{L_{25}-R_{25}}\right) x_{25}+I\left((8,10,1,1)_{L_{34}-R_{34}}\right) x_{34} \\
+I\left((10,12,1,2)_{L_{35}-R_{35}}\right) x_{35}+I\left((4,6,2,2)_{L_{45}-R_{45}}\right) x_{45}
\end{array}\right)
$$


subject to

$$
\begin{aligned}
& x_{12}+x_{13}=10 \\
& x_{24}+x_{25}-x_{12}=0 \\
& x_{34}+x_{35}-x_{13}=20 \\
& x_{45}-x_{24}-x_{34}=-15 \\
& -x_{25}-x_{35}-x_{45}=-15 \\
& 20-20(1-\lambda)^{\frac{1}{4}} \leq x_{12} \leq 30+20(1-\lambda)^{\frac{1}{4}}, 10 \lambda \leq x_{13} \leq 30+30(1-\lambda)^{\frac{1}{4}}, \\
& 20 \lambda \leq x_{24} \leq 80-20 \lambda, 10-10(1-\lambda)^{\frac{1}{2}} \leq x_{25} \leq 50-10 \lambda, \\
& 30-30(1-\lambda)^{\frac{1}{4}} \leq x_{34} \leq 60+30(1-\lambda)^{\frac{1}{2}}, 25-25(1-\lambda)^{\frac{1}{2}} \leq x_{35} \leq 50 \\
& +25(1-\lambda)^{\frac{1}{4}}, 50-50(1-\lambda)^{\frac{1}{4}} \leq x_{45} \leq 95-35 \lambda \text { and } x_{i j} \geq 0 . \\
&
\end{aligned}
$$

where, $\lambda \in[0,1]$.

Step 3: Putting the values of $I\left(\tilde{c}_{i j}\right)$, obtained by using Yager's ranking approach discussed in section 2.2, the crisp linear programming problem, obtained in Step 2, may be written as:

Minimize $\left(3.1 x_{12}+5.15 x_{13}+8.5 x_{24}+12.5 x_{25}+9 x_{34}+11.3 x_{35}+4.886 x_{45}\right)$ subject to

$$
\begin{aligned}
& x_{12}+x_{13}=10 \\
& x_{24}+x_{25}-x_{12}=0 \\
& x_{34}+x_{35}-x_{13}=20 \\
& x_{45}-x_{24}-x_{34}=-15 \\
& -x_{25}-x_{35}-x_{45}=-15 \\
& 20-20(1-\lambda)^{\frac{1}{4}} \leq x_{12} \leq 30+20(1-\lambda)^{\frac{1}{4}}, 10 \lambda \leq x_{13} \leq 30+30(1-\lambda)^{\frac{1}{4}}, \\
& 20 \lambda \leq x_{24} \leq 80-20 \lambda, 10-10(1-\lambda)^{\frac{1}{2}} \leq x_{25} \leq 50-10 \lambda, \\
& 30-30(1-\lambda)^{\frac{1}{4}} \leq x_{34} \leq 60+30(1-\lambda)^{\frac{1}{2}}, 25-25(1-\lambda)^{\frac{1}{2}} \leq x_{35} \leq 50 \\
& +25(1-\lambda)^{\frac{1}{4}}, 50-50(1-\lambda)^{\frac{1}{4}} \leq x_{45} \leq 95-35 \lambda \text { and } x_{i j} \geq 0 .
\end{aligned}
$$

where, $\lambda \in[0,1]$.

Step 4: The optimal solution of the crisp linear programming problem, obtained in Step 3 i.e., the optimal solution of chosen single-objective fuzzy MCF problem, for $\lambda=0$ is $x_{12}=10, x_{24}=10, x_{34}=5, x_{35}=15$ and rest all $x_{i j}$ are zero and the optimal value, representing, the Yager's ranking index corresponding to minimum fuzzy cost, is 330.50 .

7.1b Solution of the chosen single objective fuzzy MCF problem: Repeating all the steps of the proposed method, described in section 7.1a, the optimal solution of the single-objective fuzzy MCF problem, chosen in Case (ii) of Example 4.2, for $\lambda=0$ is $x_{12}=10, x_{25}=10, \quad x_{34}=$ $15, x_{35}=5$ and rest all $x_{i j}$ are zero and Yager's ranking index corresponding to the minimum fuzzy time is 278.30 .

7.1c Solution of the chosen multi-objective fuzzy MCF problem: The multi-objective fuzzy MCF problem, chosen in Case (iii) of Example 5.1, may be solved by using the following steps of the proposed method: 
Step 1: The fuzzy linear programming formulation of multi-objective fuzzy MCF problem is:

$$
\begin{aligned}
& \operatorname{Minimize}\left(\begin{array}{l}
(2,3,1,2)_{L_{12}-R_{12}} x_{12} \oplus(5,5,1,1)_{L_{13}-R_{13}} x_{13} \oplus(7,8,2,5)_{L_{24}-R_{24}} x_{24} \\
\oplus(10,15,1,1)_{L_{25}-R_{25}} x_{25} \oplus(8,10,1,1)_{L_{34}-R_{34}} x_{34} \\
\oplus(10,12,1,2)_{L_{35}-R_{35}} x_{35} \oplus(4,6,2,2)_{L_{45}-R_{45}} x_{45}
\end{array}\right) .
\end{aligned}
$$

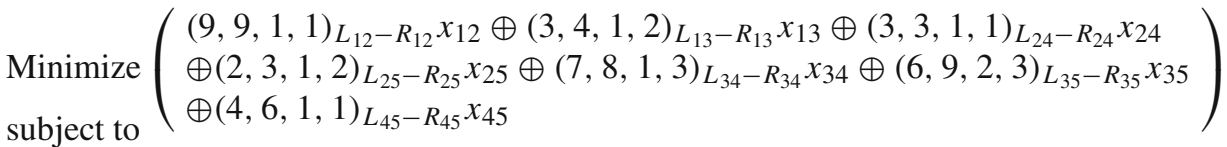

$$
\begin{aligned}
& x_{12}+x_{13}=10 \\
& x_{24}+x_{25}-x_{12}=0 \\
& x_{34}+x_{35}-x_{13}=20 \\
& x_{45}-x_{24}-x_{34}=-15 \\
& -x_{25}-x_{35}-x_{45}=-15 \\
& x_{12} \in(20,30,20,20)_{L_{12}-R_{12}}, x_{13} \in(10,30,10,30)_{L_{13}-R_{13}}, x_{24} \\
& \in(20,60,20,20)_{L_{24}-R_{24}} \\
& x_{25} \in(10,40,10,10)_{L_{25}-R_{25}} x_{34} \in(30,60,30,30)_{L_{34}-R_{34}}, x_{35} \\
& \in(25,50,25,25)_{L_{35}-R_{35}} \\
& x_{45} \in(50,60,50,35)_{L_{45}-R_{45}} .
\end{aligned}
$$

Step 2: The fuzzy multi-objective linear programming problem, obtained in Step 1, may be converted into the following crisp multi-objective linear programming problem:

$$
\begin{aligned}
& \operatorname{Minimize}\left(\begin{array}{l}
I\left((2,3,1,2)_{L_{12}-R_{12}}\right) x_{12}+I\left((5,5,1,1)_{L_{13}-R_{13}}\right) x_{13} \\
+I\left((7,8,2,5)_{L_{24}-R_{24}}\right) x_{24}+I\left((10,15,1,1) L_{25}-R_{25}\right) x_{25} \\
+I\left((8,10,1,1)_{L_{34}-R_{34}}\right) x_{34}+I\left((10,12,1,2)_{L_{35}}-R_{35}\right) x_{35} \\
+I\left((4,6,2,2)_{L_{45}-R_{45}}\right) x_{45}
\end{array}\right) \\
& \operatorname{Minimize}\left(\begin{array}{l}
I\left((9,9,1,1)_{L_{12}-R_{12}}\right) x_{12}+I\left((3,4,1,2)_{L_{13}-R_{13}}\right) x_{13} \\
+I\left((3,3,1,1)_{L_{24}-R_{24}}\right) x_{24}+I\left((2,3,1,2)_{L_{25}-R_{25}}\right) \\
x_{25}+I\left((7,8,1,3)_{L_{34}-R_{34}}\right) x_{34}+I\left((6,9,2,3)_{L_{35}-R_{35}}\right) x_{35} \\
+I\left((4,6,1,1)_{L_{45}-R_{45}}\right) x_{45}
\end{array}\right)
\end{aligned}
$$

subject to

$$
\begin{aligned}
& x_{12}+x_{13}=10 \\
& x_{24}+x_{25}-x_{12}=0 \\
& x_{34}+x_{35}-x_{13}=20 \\
& x_{45}-x_{24}-x_{34}=-15 \\
& -x_{25}-x_{35}-x_{45}=-15 \\
& 20-20(1-\lambda)^{\frac{1}{4}} \leq x_{12} \leq 30+20(1-\lambda)^{\frac{1}{4}}, 10 \lambda \leq x_{13} \leq 30+30(1-\lambda)^{\frac{1}{4}}, \\
& 20 \lambda \leq x_{24} \leq 80-20 \lambda, 10-10(1-\lambda)^{\frac{1}{2}} \leq x_{25} \leq 50-10 \lambda, \\
& 30-30(1-\lambda)^{\frac{1}{4}} \leq x_{34} \leq 60+30(1-\lambda)^{\frac{1}{2}}, 25-25(1-\lambda)^{\frac{1}{2}} \leq x_{35} \leq \\
& \quad 50+25(1-\lambda)^{\frac{1}{4}}, \\
& 50-50(1-\lambda)^{\frac{1}{4}} \leq x_{45} \leq 95-35 \lambda \text { and } x_{i j} \geq 0 .
\end{aligned}
$$

where, $\lambda \in[0,1]$. 
Step 3: Putting the values of $I\left(\tilde{c}_{i j}\right)$ and $I\left(\tilde{t}_{i j}\right)$, obtained by using Yager's ranking approach discussed in section 2.2, the crisp multi-objective linear programming problem, obtained in Step 2, may be written as:

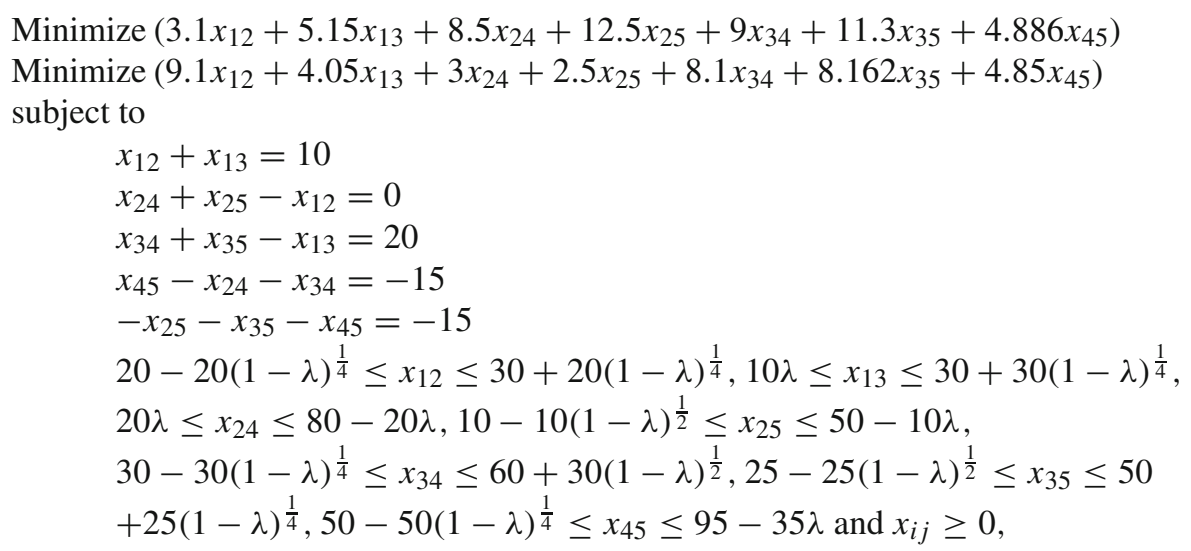

where, $\lambda \in[0,1]$.

Step 4: Using the fuzzy programming technique, discussed in section 5, the optimal compromise solution for $\lambda=0$ is $x_{12}=10, x_{24}=5.01, x_{25}=4.99, x_{34}=9.99, x_{35}=10.01$ and rest all $x_{i j}$ are zero.

Step 5: Putting the values of $x_{i j}$ in $3.1 x_{12}+5.15 x_{13}+8.5 x_{24}+12.5 x_{25}+9 x_{34}+11.3 x_{35}+$ $4.886 x_{45}$ and $9.1 x_{12}+4.05 x_{13}+3 x_{24}+2.5 x_{25}+8.1 x_{34}+8.162 x_{35}+4.85 x_{45}$ the Yager's ranking index corresponding to the minimum fuzzy cost and minimum fuzzy time are 338.983 and 281.125, respectively.

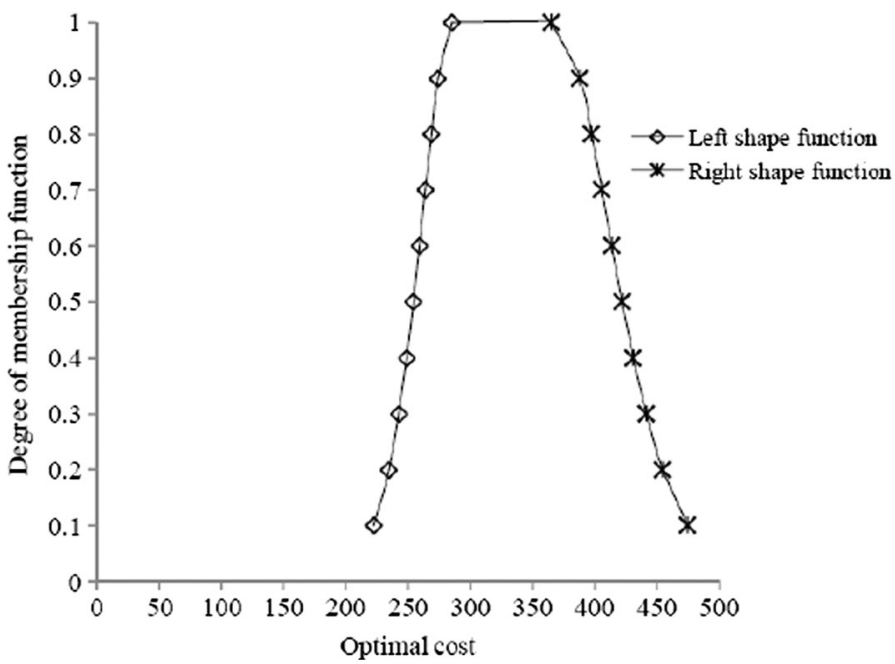

Figure 2. Membership functions of the $L-R$ fuzzy number representing the minimum fuzzy cost. 


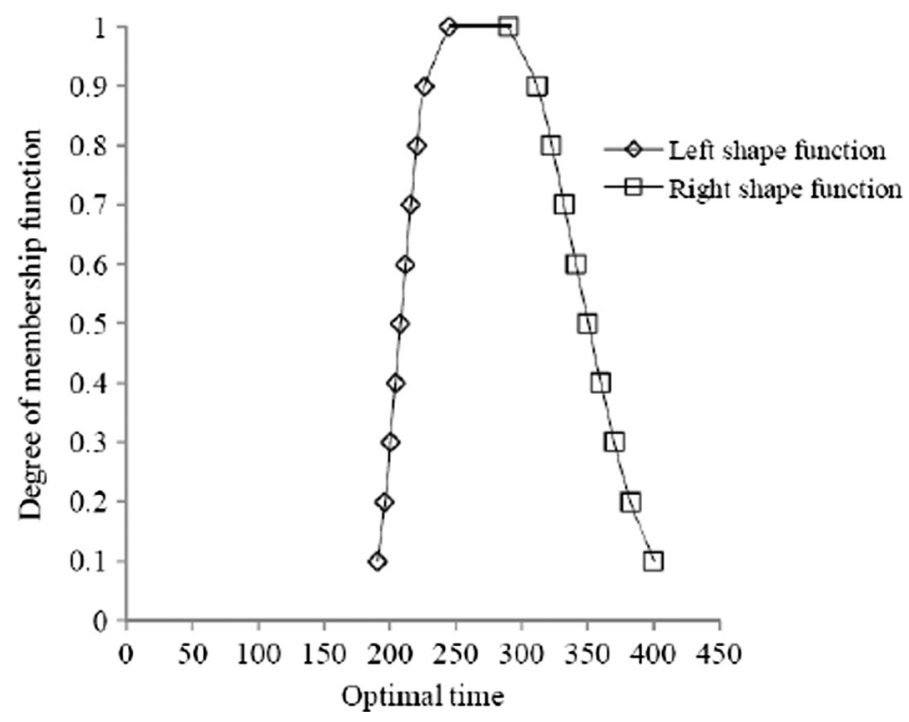

Figure 3. Membership functions of the $L-R$ fuzzy number representing the minimum fuzzy time.

Membership functions of the $L-R$ fuzzy numbers representing the minimum fuzzy cost and minimum fuzzy time are shown in figures 2 and 3 , respectively.

\section{Results and discussion}

To show the advantages of the proposed method over existing methods (Shih \& Lee 1999; Liu \& Kao 2004; Ghatee et al 2009) single and multi-objective fuzzy MCF problems, chosen in section 4 which cannot be solved by the existing methods (Shih \& Lee 1999; Liu \& Kao 2004; Ghatee et al 2009), are solved in section 7. The obtained results are shown in tables 5 and 6.

It is obvious from the results shown in tables 5 and 6, that the fuzzy MCF problem, chosen in Example 4.1, may be solved by using both the existing methods (Shih \& Lee 1999; Ghatee et al 2009) and the proposed methods and the results of existing method (Liu \& Kao 2004) and the proposed method are same. While, the fuzzy MCF problems, chosen in Case (i) and Case (ii), Case (iii) of Examples 4.2 may be solved only by using the proposed method i.e., it is not

Table 5. Results of existing and proposed methods for solving single objective fuzzy MCF problems.

\begin{tabular}{lccc}
\hline $\begin{array}{l}\text { Single objective } \\
\text { fuzzy MCF problem }\end{array}$ & $\begin{array}{c}\text { Existing method } \\
(\text { Shih \& Lee 1999) }\end{array}$ & $\begin{array}{c}\text { Existing method } \\
\text { (Ghatee } \text { et al 2009) }\end{array}$ & $\begin{array}{c}\text { Proposed } \\
\text { method }\end{array}$ \\
\hline $\begin{array}{l}\text { Example 4.1 } \\
\text { Case (i). of } \\
\text { Example 4.2 }\end{array}$ & $\begin{array}{c}(6805,5620,10255) \\
\text { Not applicable }\end{array}$ & $\begin{array}{c}(6605,5365,11420) \\
\text { Not applicable }\end{array}$ & $(6805,5620,10255)$ \\
$\begin{array}{l}\text { Case (ii). of } \\
\text { Example 4.2 }\end{array}$ & Not applicable & Not applicable & $\sum_{(i, j) \in A} I\left(\tilde{c}_{i j}\right) x_{i j}=330.50$ \\
& & $\sum_{(i, j) \in A} I\left(\tilde{t}_{i j}\right) x_{i j}=278.30$ \\
\hline
\end{tabular}


Table 6. Results of existing and proposed methods for solving multi-objective fuzzy MCF problems.

\begin{tabular}{lcccc}
\hline $\begin{array}{l}\text { Multi-objective } \\
\text { fuzzy MCF } \\
\text { problem }\end{array}$ & $\begin{array}{l}\text { Existing method } \\
\text { (Shih \& } \\
\text { Lee 1999) }\end{array}$ & $\begin{array}{l}\text { Existing method } \\
\text { Liu \& } \\
\text { Kao 2004) }\end{array}$ & $\begin{array}{l}\text { Existing method } \\
\text { (Ghatee } \\
\text { et al 2009) }\end{array}$ & $\begin{array}{c}\text { Proposed } \\
\text { method }\end{array}$ \\
\hline Case (iii) of Example 4.2 & $\begin{array}{c}\text { Not } \\
\text { applicable }\end{array}$ & $\begin{array}{c}\text { Not } \\
\text { applicable }\end{array}$ & $\begin{array}{c}\text { Not } \\
\text { applicable }\end{array}$ & $\begin{array}{c}\sum_{(i, j) \in A} I\left(\tilde{c}_{i j}\right) x_{i j}=338.983 \\
\text { and } \\
\sum_{(i, j) \in A} I\left(\tilde{t}_{i j}\right) x_{i j}=281.125\end{array}$ \\
\hline
\end{tabular}

possible to solve these problems by using any of the existing methods (Shih \& Lee 1999; Liu \& Kao 2004; Ghatee et al 2009). On the basis of above discussion, it may be suggested that it is better to use the proposed method to solve single and multi-objective fuzzy MCF problems instead of the existing methods (Shih \& Lee 1999; Liu \& Kao 2004; Ghatee et al 2009).

\section{Conclusions}

A new method is proposed to solve single and multi-objective fuzzy MCF problems. The main advantage of the proposed method over the existing methods (Shih \& Lee 1999; Liu \& Kao 2004; Ghatee et al 2009) is that there are several single and multi-objective fuzzy MCF problems which cannot be solved by using the existing methods but can be solved by using the proposed method. To illustrate the proposed method, single and multi-objective fuzzy MCF problems are solved and the obtained results are discussed.

\section{Acknowledgements}

The authors would like to thank the Editor-in-Chief and anonymous referees for various suggestions which have led to an improvement in both the quality and clarity of the paper. I, Dr. Amit Kumar, want to acknowledge the adolescent inner blessings of Mehar. I believe that Mehar is an angel for me and without Mehar's blessing it was not possible to think the idea proposed in this paper. Mehar is a lovely daughter of Parmpreet Kaur (Research Scholar under my supervision).

\section{References}

Ahuja R K, Magnanti T L and Orlin J B 1993 Network flows. Englewood Cliffs: Prentice-Hall

Chanas S and Kuchta D 1998 Fuzzy integer transportation problem. Fuzzy Set Syst. 98: 291-298

Chen S P 2007 Analysis of critical paths in a project network with fuzzy activity times. Eur. J. Oper. Res. 183: 442-459

Chen S P and Hsueh Y J 2008 A simple approach to fuzzy critical path analysis in project networks. Appl. Math. Model 32: 1289-1297

Ching K L and Cheng C H 2009 A novel general approach to evaluating the PCBA for components with different membership function. Appl. Soft Comp. 9: 1044-1056

Dubois D and Prade H 1980 Fuzzy sets and systems: Theory and applications. New York: Academic Press

Ghatee M and Hashemi S M 2007 Ranking function-based solutions of fully fuzzified minimal cost flow problem. Inform. Sci. 177: 4271-4294

Ghatee M and Hashemi S M 2008 Generalized minimal cost flow problem in fuzzy nature: an application in bus network planning problem. Appl. Math. Model 32: 2490-2508

Ghatee M and Hashemi S M 2009 Application of fuzzy minimum cost flow problems two network design under uncertainty. Fuzzy Set Syst. 160: 3263-3289 
Ghatee M, Hashemi S M, Hashemi B and Dehghan M 2008 Solution and duality of imprecise network problems. Comput. Math. Appl. 55: 2767-2790

Ghatee M, Hashemi S M, Zarepisheh M and Khorram E 2009 Preemptive priority-based algorithms for fuzzy minimal cost flow problem: An application in hazardous materials transportation. Comput. Ind. Eng. 57: 341-354

Hamacher H W, Pedersen C R and Ruzika S 2007 Multiple objective minimum cost flow problems: A review. Eur. J. Oper. Res. 176: 1404-1422

Kumar A, Yadav S P and Kumar S 2008 Fuzzy system reliability using different types of vague sets. Int. J. Appl. Sci. Eng. 6: 71-83

Liu S T and Kao C 2004 Network flow problems with fuzzy arc lengths. IEEE T. Syst. Man. Cy. B 34: 765-769

Mon D L and Cheng C H 1994 Fuzzy system reliability analysis for components with different membership functions. Fuzzy Set Syst. 64: 145-157

Shih H S and Lee E S Fuzzy multi-level minimum cost flow problems. Fuzzy Set Syst. 107: 159-176

Verma R, Biswal M P and Biswas A 1997 Fuzzy programming technique to solve multi-objective transportation problems with some non-linear membership functions. Fuzzy Set Syst. 91: 37-43

Yager R R 1981 A procedure for ordering fuzzy subsets of the unit interval. Inform. Sci. 24: 143-161

Zadeh L A 1965 Fuzzy sets. Inform. Control 8: 338-353

Zareei A, Zaerpour F, Bagherpour M, Noora A and Vencheh A H 2011 A new approach for solving fuzzy critical path problem using analysis of events. Expert Syst. Appl. 38: 87-93 